\title{
Desenvolvimento e análise do projeto do sistema de direção para um protótipo Baja
} SAE

\section{Development and analysis of the steering system design for a prototype Baja SAE}

\author{
1 Gabriel de Souza Faria Montanari gabrielmontanari@id.uff.br \\ 1 Lucas da Silva Reis de Lima \\ 1 Luiz Claudio da Cruz Almeida \\ 1 Lucca Lazaroni Sacchetto \\ 2 Maria Carolina dos Santos Freitas
}

1 Estudante de Engenharia Mecânica. Universidade Federal Fluminense - Campus Escola de Engenharia Industrial e Metalúrgica de Volta Redonda.

2 Doutora em Engenharia Metalúrgica, Mestre em Engenharia Metalúrgica e Engenheira Mecânica pela Universidade Federal Fluminense.

\section{Resumo}

Os protótipos Baja SAE são projetados e fabricados por acadêmicos que participam anualmente de competições realizadas pela SAE (Society of Automotive Engineers) e possuem o intuito de ajudar no desenvolvimento técnico e profissional dos estudantes, além de buscar inovações para o setor automotivo. Desta forma, o objetivo deste artigo é desenvolver e analisar um sistema de direção que atenda aos esforços requisitados em tais competições. Para isso, utilizando-se a geometria de Ackerman, foram determinados os parâmetros para iniciação no SolidWorks ${ }^{\circledR}$. Em desenho 2D, foi encontrado o curso da cremalheira e a relação volante/pinhão, que determinou o ângulo de giro do volante e o raio de giro do veículo. Com a utilização de equações, foi determinado o diâmetro primitivo do pinhão, a força horizontal que o sistema sofrerá e o torque gerado pelo piloto, possibilitando o avanço para o desenho em 3D dos componentes da caixa de direção e suas análises. Uma vez concluído que as peças suportam as condições submetidas, torna-se possível a fabricação e utilização delas. Para apresentação dos resultados, obteve-se auxílio de softwares de simulações, como MSC ADAMS $\AA^{\text {e e Ansys }}{ }^{\circledR}$, de modo a permitir a otimização de projetos nos aspectos de desempenho, segurança e conforto.

Palavras-chave:

Direção. Cremalheira. Baja SAE. Simulações.

\begin{abstract}
The Baja SAE prototypes are designed and manufactured by academics who participate annually in competitions accomplished by SAE (Society of Automotive Engineers) and are intended to help in the technical and professional development of the students, in addition to seeking innovations for the automotive sector. Thus, the objective of this article is to develop and analyze a steering system that meets the efforts required in such competitions. For that, using Ackerman's geometry, the parameters for initiation into SolidWorks ${ }^{\circledR}$ were determined. In $2 D$ design was found the rack stroke and the relation steering wheel / pinion ratio, which determined the steering wheel turning angle and the turning radius of the vehicle. Using equations, it was determined the primitive diameter of the pinion, the horizontal force that the system will suffer and the torque generated by the pilot, allowing the advance to the $3 D$ drawing of the components of the steering box and their analysis. Once it is concluded that the parts support the submitted conditions, it becomes possible the manufacturing and the use of them. To present the results, the software simulations aid was obtained, such as MSC ADAMS ${ }^{\circledR}$ and Ansys ${ }^{\circledR}$, in order to permit the optimization of projects in performance, safety and comfort aspects.
\end{abstract}

\section{Keywords:}

Steering. Rack. Baja SAE. Simulations.

Como você deve citar?

MONTANARI, Gabriel de Souza Faria et al. Desenvolvimento e análise do projeto do sistema de direção para um protótipo Baja SAE. Cadernos UniFOA, Volta Redonda (RJ), v. 16, n. 47, p. 07-15, dez, 2021 
O projeto Baja SAE foi criado nos Estados Unidos, na Universidade da Carolina do Sul, pelo Dr. John F. Stevens. Sua primeira competição aconteceu no ano de 1976; em 1991, foi dado início às atividades do Baja no Brasil e, em 1994, lançou-se o Projeto Baja SAE Brasil. Este, por sua vez, possui o intuito de fazer com que os alunos de engenharia coloquem em prática os conhecimentos adquiridos em sala de aula ao desenvolver e validar seus projetos em competições de alto nível, com provas como Suspension e Enduro, as quais consistem em obstáculos como troncos, canaletas, valas, barrancos, entre outros desafios, em que os veículos e seus componentes são submetidos a forças e tensões críticas. Dessa forma, seu objetivo de capacitar o estudante para o mercado de trabalho é satisfeito, assim como o desenvolvimento de pesquisas, novas tecnologias e inovações para o setor automobilístico.

Fundada em 2005, a Equipe Forja Baja é dividida em subdivisões com a finalidade de otimizar a elaboração do protótipo. A ramificação de Suspensão e Direção é responsável pelo projeto de todo o sistema de suspensão, sendo este incumbido por manter o contato das rodas com o solo, absorvendo os impactos do terreno e gerando conforto para o piloto, mesmo quando se deve transpor obstáculos. Por sua vez, o sistema de direção, segundo Gillespie (1992), pode ser definido por possuir a função de dirigir as rodas dianteiras em resposta às entradas de comando do motorista, a fim de fornecer controle direcional global do veículo.

Existem inúmeros sistemas de suspensão, como Duplo A, MacPherson, Semi-Trailing, Trailing-Arm, entre outros. A escolha do tipo é muito importante, visto que cada um limita de diferentes maneiras a direção, como espaço, por exemplo. 0 modelo selecionado para o protótipo foi a suspensão Duplo $A$, que é caracterizada por dois braços de controle com formato de letra A. Além de ocupar pouco espaço e ser vantajosa em veículos com estruturas específicas para montagem da suspensão, apresenta também muitas possibilidades de trabalho em variação de cambagem, convergência e boa absorção de forças, sendo uma das mais utilizadas em veículos Baja SAE.

Portanto, o objetivo deste trabalho é elaborar um sistema de direção para um protótipo Baja SAE, através de simulações em softwares especializados, a fim de evitar que ocorram quebras ou empenamentos, como já acontecido anteriormente na equipe. 0 enfoque do trabalho será no estudo das forças a que esse sistema é submetido, análise da quebra dos componentes que sofrem mais esforços, como cremalheira e caixa de direção, visto que os demais itens recebem menores tensões, além de redução de massa do sistema como um todo, mantendo a confiabilidade do projeto, conforme as restrições impostas pelo Regulamento Administrativo e Técnico Baja SAE Brasil (SAE BRASIL, 2019).

\section{MATERIAIS E MÉTODOS}

De acordo com Rosa (2010, p.3), uma sensação agradável de direção é proporcionada escolhendo-se um mecanismo cuja geometria não permita conflitos entre os deslocamentos horizontais da direção e os movimentos verticais da suspensão. Para a determinação de valores iniciais de projeto para o desenho da direção, é usual considerar o movimento do veículo em plano horizontal somente e, para as curvas, um raio de curvatura constante. Isso permite uma condição de análise de dirigibilidade em regime permanente com premissas simplificadas, dada a definição de valores iniciais para as variáveis geométricas.

A geometria de Ackerman (Figura 1) é definida por Peetz (2018, p.21) como um trapezoide que produz no conjunto pneu-roda ângulos de esterçamento, devido a isso, a curvatura da roda interna é maior que o da externa. Essa geometria garante uma melhor relação entre os enfoques de giro dos conjuntos e determina o centro de curvatura do veículo sem que haja arraste dos pneus. Esses arcos são concêntricos, 
mas de raios diferentes e isso implica em ângulos de esterçamento distintos. Caso a geometria não seja obedecida, os pneus entrarão em conflito, contribuindo com instabilidade e desgaste primitivo.

Figura 1: Explicação da Geometria de Ackerman

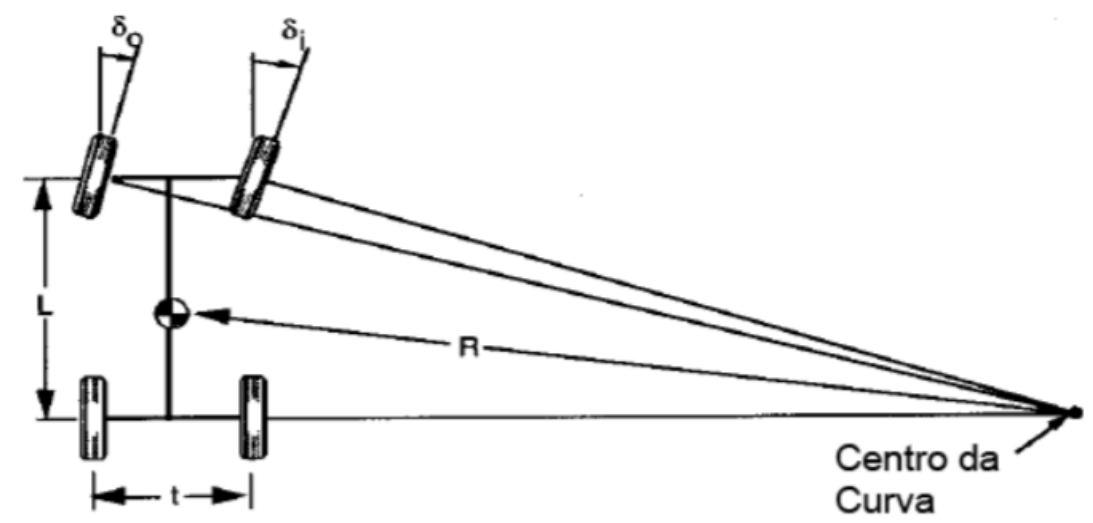

Fonte: Gillespie (1992)

Onde:

R: Distância do centro da curva até o CG;

L: Entre eixos do protótipo;

t: Distância entre as rodas (Bitola);

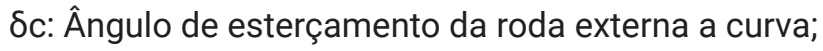

ठi: Ângulo de esterçamento da roda interna a curva.

Além da geometria de Ackerman, para a construção do sistema de direção, são necessários outros dados, como torque e força radial no volante, que podem ser vistos na Tabela 1. Essas condições de contorno são essenciais para que seja realizado o dimensionamento adequado do projeto. 
Tabela 1: Tabela de parâmetros para dimensionamento

\begin{tabular}{ccc}
\hline Condição de contorno & Carregamento estático & Fonte de dados \\
\hline Força vertical (N) & Suspensão dianteira & \\
\hline Força longitudinal (N) & 10.000 & Multicorpos \\
\hline Força vertical (N) & 1076 & Análise de falha \\
\hline Força longitudinal (N) & Suspensão traseira & \\
\hline Torque máximo no volante (N.mm) & 10.000 & Multicorpos \\
\hline Força radial no volante (N) & 100.000 & Artigo \\
\hline
\end{tabular}

Fonte: Autoria própria (2020)

De acordo com Fox (2010, p.3), com base em testes e validações, o torque exercido no volante pelo motorista possui valores entre $3400 \mathrm{~N} . \mathrm{mm}-88000 \mathrm{~N} . \mathrm{mm}$, logo, obedecendo a um fator de segurança pré-determinado, foi encontrado um valor de 100.000 N.mm como valor para ser usado como condição de contorno. Ainda segundo Fox (2010, p.4), a força radial no volante, apesar de ser relativamente pequena quando o carro está em movimento, deve ser considerada e aguentar, no mínimo, 60N.

Além desses valores, em prol de um melhor dimensionamento, simulações são realizadas, com o objetivo de se obter os esforços em componentes específicos, como o pinhão e cremalheira, que podem ser vistos na Figura 2.

Figura 2: Vista Explodida com os componentes da direção

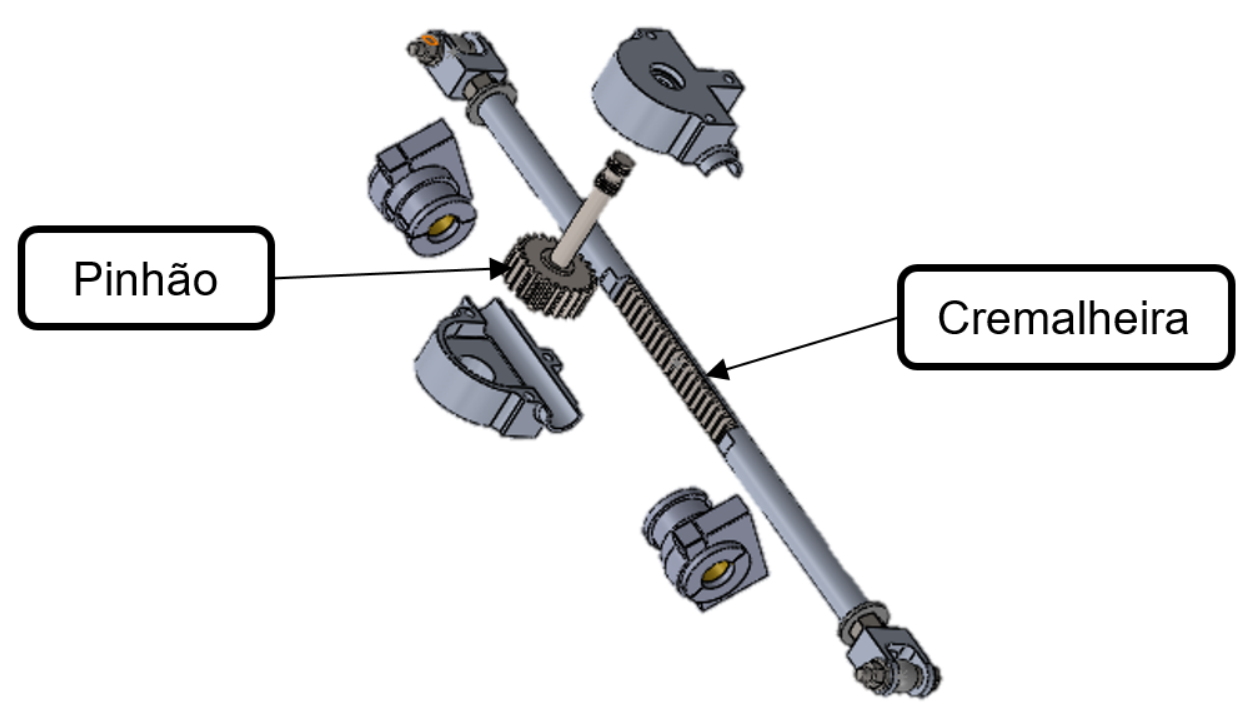

Fonte: Autoria própria (2020) 
Para o dimensionamento do conjunto pinhão/cremalheira, foi necessário estipular alguns parâmetros preliminares, sendo estes, o módulo das engrenagens, ângulo de pressão, curso linear da cremalheira para cada lado e número de dentes que garanta o engrenamento do par.

Tabela 2: Parâmetros Preliminares

\begin{tabular}{ll}
\hline Módulo de engrenagem (mm) & 2,5 \\
\hline Ângulo de pressão (Graus) & 20 \\
\hline Curso da cremalheira (mm) & 35 \\
\hline Número mínimo de dentes & 20 \\
\hline Número de dentes & 24 \\
\hline
\end{tabular}

Fonte: Autoria própria, 2020

Tendo definido tais parâmetros, obteve-se um ângulo de 90 graus de giro do volante para cada lado, próximo do que era objetivado. Com o intuito de dimensionar estruturalmente, foi definido, experimentalmente, através do uso de um torquímetro, o maior torque realizado pelos pilotos da equipe. Assim, foi definida também a largura de face do pinhão, para que seu comprimento fosse igual ao diâmetro do eixo da cremalheira e não comprometesse a sua integridade estrutural.

Através da simulação de colisão (Figura 3), sendo estimado valores de velocidade de choque, é possível se obter parâmetros de referência dinâmicos (Tabela 3), como força de impacto e deformação ocorrida antes da quebra de componentes específicos.

Tabela 3: Tabela com valores dinâmicos

\begin{tabular}{ll}
\hline \multicolumn{2}{c}{ Condições de contorno } \\
\hline Velocidade de impacto $(\mathrm{km} / \mathrm{h})$ & 10 \\
\hline Deformação na cremalheira $(\mathrm{mm})$ & 5 \\
\hline Deformação no braço de direção $(\mathrm{mm})$ & 10 \\
\hline
\end{tabular}

Fonte: Autoria própria (2020)

Com os parâmetros estabelecidos na Tabela 1 e utilizando-se os valores das Tabelas 2 e 3 , torna-se possível, segundo Brito (2017, p.4), elaborar um modelo matemático para realizar a simulação, com o intuito de analisar o comportamento dinâmico do protótipo. Assim, foi desenvolvido um modelo para avaliar a dinâmica do veículo, utilizando-se o software MSC ADAMS ${ }^{\circledast}$ (Figura 3). A condição crítica escolhida foi uma colisão frontal no máximo esterçamento em um obstáculo. Além disso, a velocidade adotada para tal situação foi de $10 \mathrm{~km} / \mathrm{h}$, cujo controle é feito pelo próprio usuário. 
Figura 3: Simulação de Impacto

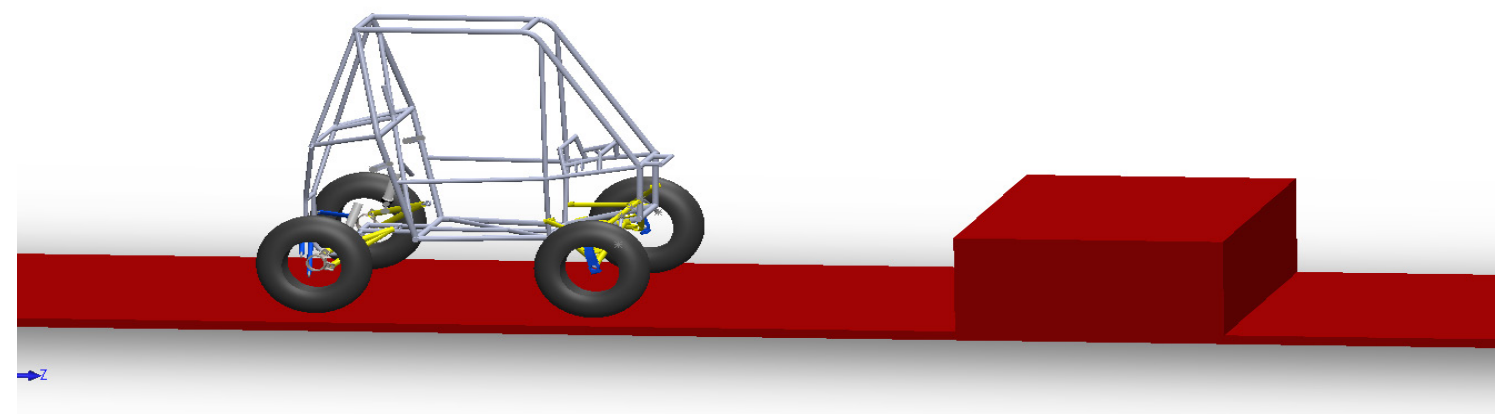

Fonte: Autoria própria (2020)

A partir dessa simulação, foi possível adquirir as cargas atuantes em cada peça em situações críticas em que o protótipo é submetido. Para dimensionar os componentes do sistema de direção, foi utilizada a maior força aquisitada na análise.

Para garantir o constante alinhamento da cremalheira com as buchas da caixa e guias da direção, primeiramente foi projetada uma estrutura bipartida, tampa e base da caixa de direção, como observado nas Figuras 4 e 5. Todavia, o alinhamento já seria garantido somente com a parte inferior, tendo em vista que é, a partir dele, que há o contato com o chassi. Assim, foi possível reduzir cerca de $30 \%$ do peso do conjunto.

Figura 4: Caixa de Direção Antiga

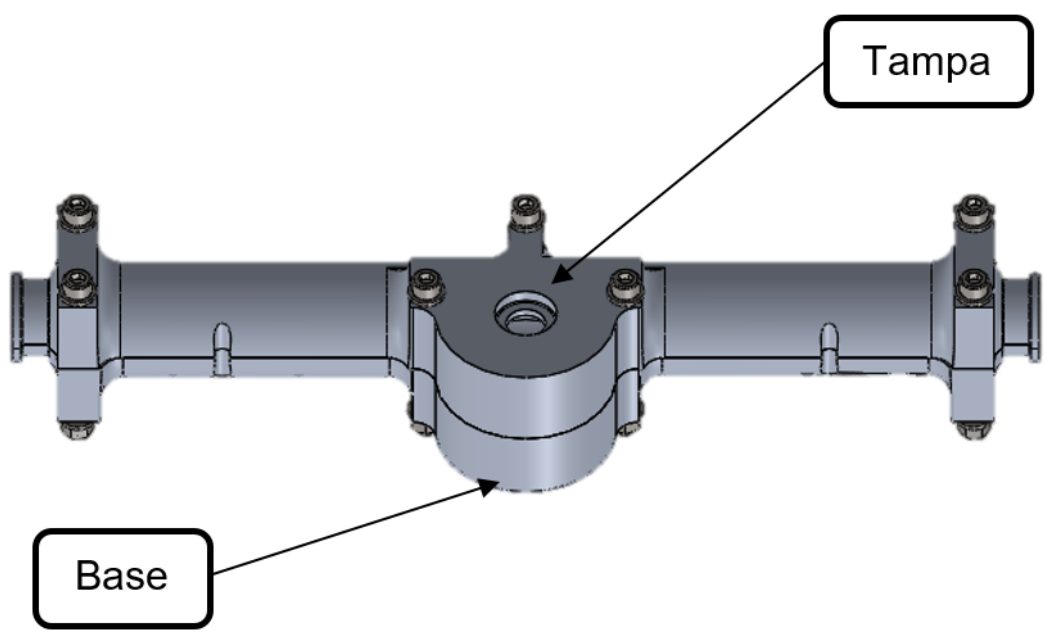

Fonte: Autoria própria (2020) 
Figura 5: Projeto caixa de direção

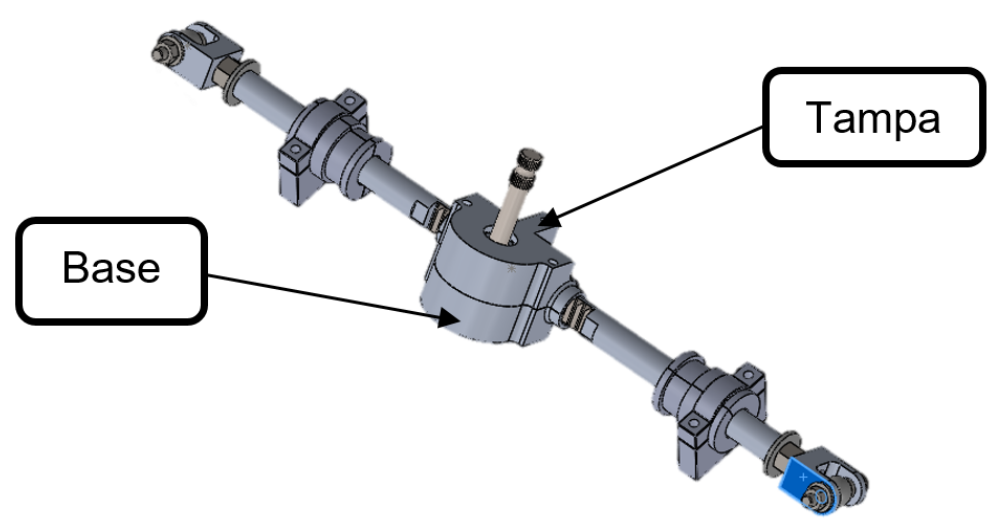

Fonte: Autoria própria (2020)

Além de estudos em componentes, como o pinhão e cremalheira, também foram realizadas simulações em Ansys ${ }^{\circledast}$, na caixa de direção em si, com base nas condições de contorno da Tabela 4, com o intuito de garantir a integridade de itens essenciais anteriormente citados, como também, em caso de quebra de componentes internos, como pinhão e cremalheira, seja garantida a segurança do piloto da equipe.

Tabela 4: Condições de contorno para simulação da caixa de direção

\begin{tabular}{cc}
\hline \multicolumn{2}{c}{ Definição } \\
\hline Tipo & Força Remota $(\mathrm{N})$ \\
\hline Eixo X & 7547 \\
\hline Eixo $Y$ & 0 \\
\hline Eixo $Z$ & -2581 \\
\hline
\end{tabular}

Fonte: Autoria própria (2020)

\section{RESULTADOS E DISCUSSÃO}

É possível observar, a partir da Figura 6, o resultado da simulação feita no software MSC ADAMS ${ }^{\circledR}$, de Força x Tempo, em que é apresentado o pico de força nos braços de direção, no momento do impacto do protótipo com as rodas esterçadas no obstáculo. Pelas forças aquisitadas, é observada flambagem no corpo da cremalheira, contudo não seria suficiente para que ocorresse a quebra do componente, apresentando conformidade e semelhança com relação a outros estudos sobre o tema, sendo assim, garantida a segurança do piloto. 
Figura 6: Força atuante na cremalheira durante a simulação

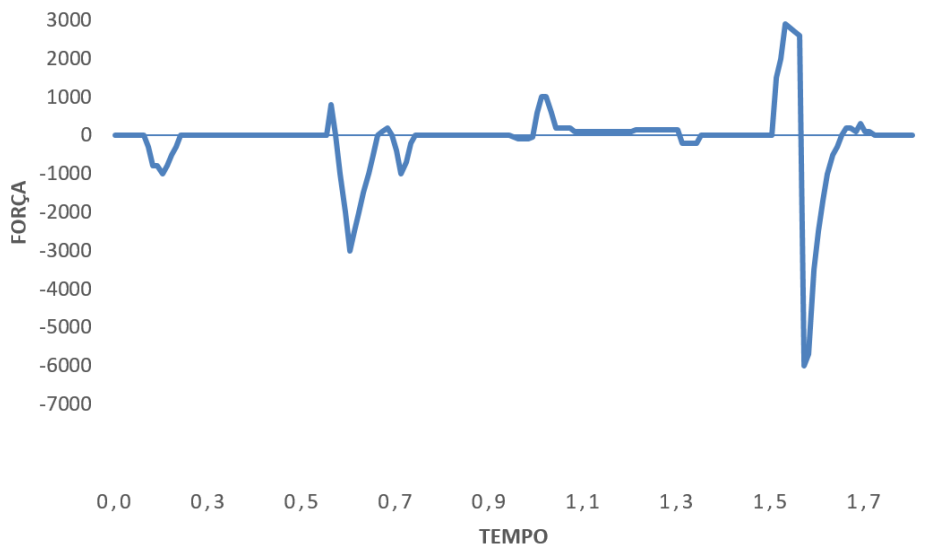

Fonte: Autoria própria (2020)

Como pode ser observado pela simulação feita na base da caixa de direção (Figura 7), mesmo sendo submetida a tensões máximas maiores do que as estipuladas possíveis, a caixa apresenta deformação com valores bem pequenos e não apresenta ruptura, logo, é possível a aplicação prática do modelo, pois, dessa forma, mesmo em caso de quebra do sistema de direção, a segurança e integridade do motorista do protótipo é garantida.

Figura 7: Simulação Estática da Caixa de Direção segundo condições de contorno

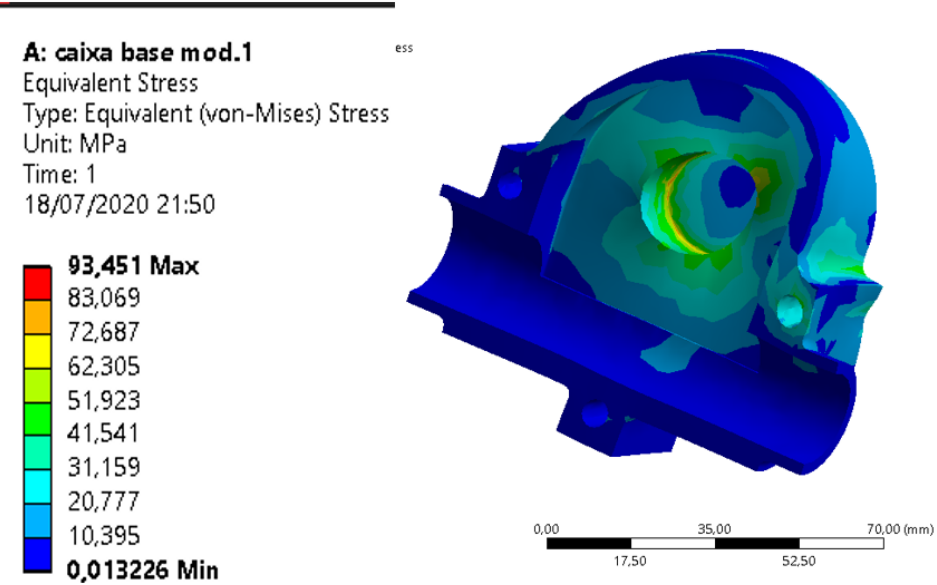

Fonte: Autoria própria, 2020. 


\section{CONSIDERAÇÕES FINAIS}

Ao analisar os resultados do estudo, é possível concluir que foram satisfatórios, tendo em vista que foram próximos do almejado. Foi possível fazer o projeto de um sistema inteiriço e que propicie maior segurança e confiabilidade para a direção, evitando quebras e rupturas. Seus componentes foram projetados com um fator de segurança associado, dentro da faixa recomendada pela literatura para tensões e forças típicas de veículos off-road, garantindo, assim, componentes seguros para o uso. Com relação à dinâmica do protótipo, todos os requisitos citados foram atingidos, como resistência à tensão máxima, obtida através da simulação de componentes críticos e redução de massa da caixa de direção. Ademais, um resultado além das expectativas foi o raio de curva do protótipo, uma vez que o atingido foi 1,8 metros e o objetivado era 2,00 metros.

\section{REFERÊNCIAS}

BRITO, Matheus Hoffmann. Desenvolvimento e dimensionamento do projeto do subsistema de direção de veículo fora de estrada. 2017. Trabalho de Conclusão de Curso (Graduação) - Curso de Engenharia Mecânica, PUC-Rio, Rio de Janeiro, 2017

FOX, Steven. Cockpit Control Forces or How Robust Do Driver Controls Really Need to Be? 2010. Monografia (Especialização) - University of Michigan, Ann Arbor, Michigan, 2010.

GILLESPIE, T.D. Fundamental of Vehicle Dynamics. 1. ed. Warrendale: Society of Automotive Engineers, 1992.

PEETZ, Renan Augusto Marchezin. Projeto de uma caixa de direção para um protótipo Baja. 2018. Trabalho de Conclusão de Curso (Graduação) - Fundação Municipal de Ensino de Piracicaba, Escola de Engenharia de Piracicaba, São Paulo, 2018.

ROSA, Manoela Tarcila Martins. Sistemas de direção. 2010. Monografia (Especialização) - Curso de Mecânica de Automóveis, Senai - Hortotec, Belo Horizonte, 2010.

SAE BRASIL. Regulamento Administrativo e Técnico Baja SAE BRASIL. São Paulo, 2019. Disponível em: https://saebrasil.org.br/programas-estudantis/baja-sae-brasil/regras-e-relatorios/. Acesso em: 20 mar. 2020. 